International Journal of Ophthalmology \& Eye Science (IJOES)

ISSN 2332-290X

\title{
Visual Evoked Potential and Glaucoma management- A Review
}

Kirstein EM

Harper's Point Eye Associates, Cincinnati, Ohio, USA.

\section{*Corresponding Author:}

Elliot M. Kirstein, OD,

Harper's Point Eye Associates, Cincinnati, Ohio, USA.

E-mail: drkirstein1@gmail.com

Received: January 27, 2015

Accepted: January 29, 2015

Published: February 04, 2015

Citation: Kirstein EM (2015) Visual Evoked Potential and Glaucoma management- A Review. Int J Ophthalmol Eye Res. S1:003, 10-13. doi: http://dx.doi.org/10.19070/2332-290X-SI01003

Copyright: Kirstein $\mathbf{E M}^{\circ}$ 2015. This is an open-access article distributed under the terms of the Creative Commons Attribution License, which permits unrestricted use, distribution and reproduction in any medium, provided the original author and source are credited.

Visual Evoked Potential (VEP) It is part of the total, gross, electrical brain activity known as the electroencephalogram and offers the potential of providing earlier and more specific objective functional testing than currently available with subjective threshold visual fields. With newer imaging methods such as Ocular Coherence Tomography (OCT), we have made significant strides in earlier detection of structural damage to the optic nerve and nerve fiber layer, while our ability to detect functional loss earlier than what we achieve via white on white threshold field analysis remains a challenge in glaucoma [1].

Primary open-angle glaucoma is described distinctly as a multifactorial optic neuropathy that is chronic and progressive with a characteristic acquired loss of optic nerve fibers. Such loss develops in the presence of characteristic subjective visual field abnormalities and manifests by cupping and atrophy of the optic disc.

Threshold visual field analysis does not selectively reveal which structures contribute to the impairment of the visual system observed in glaucoma. It has been suggested that damage to the ganglion cells and/or their axons produce glaucomatous visual field defect. VEP objectively measures the functional responses of the entire visual pathway from the anterior segment of the eye to the visual cortex and, in this context, may reliably add specific and unique information to our diagnostic protocols.

\section{The Test}

The VEP is the objective measurement of visual function monitored at the level of the occipital cortex with scalp electrodes. It is recorded with a uniform stimulus check size and a slow reversal rate throughout the field. The technology of SD-tVEP is based on a conventional pattern-reversal VEP technique [2]. A set of predetermined stimulus patterns consisting of a series of low-contrast (Lc) and high-contrast temporally modulated checkerboards is used to elicit VEP responses from the magnocellular and parvocellular pathways of the visual system. The magnocellularand parvocellular pathways can be isolated by the contrast level of the stimulus pattern. The magnocellular pathway responds to low-contrast stimulation, while the parvocellular pathway responds to high-contrast stimulation $[3,4]$. Glaucoma is characterized by progressive loss of retinal ganglion cells and their axons [5]. The electrical pulses from the ganglion cells are transmitted to the cerebral cortex via the optic nerve, optic tract, lateral geniculate nucleus, and the optic radiations. Any interruption of the transmission of these electrical pulses can be monitored using VEP [6]. Glaucoma affects the parvocellular and magnocellular cells at the same rate; however, the parvocellular cells contribute $80 \%$ of the total ganglion cell population while magnocellular cells make up only $10 \%[7,8]$. Since the ratio of healthy magnocellular cells compared to the total magnocellular - cell population approaches zero much faster than the parvocellular - cell ratio, isolation of the magnocellular - cell group by specific VEP stimuli may be beneficial in detecting early disease [6].

The pattern VEP was compared to the Octopus 2000R automated perimeter in the assessment of central visual function in chronic simple glaucoma in 90 patients ( 52 males and 38 females) in two age bands 40-60 years and 61-80 years [9]. VEP demonstrated a high detection rate $(86.7 \%)$ with a relatively low false positive rate of $7.7 \%$ ( $<<0.01)$. When the two tests were compared, absolute latency and field loss were poorly correlated but interocular differences showed much stronger correlation with the sum of field losses determined with static perimetry. This was true for both upper and lower hemifield testing. Once inter individual variability was eliminated; severity of field loss was mirrored by prolongation of VEP latency.

\section{Current Clinical Applications of VEP}

Two commercially available instruments, the Diopsys and the EvokeDx, allow clinicians to provide VEP testing with relative convenience and affordability.

The Diopsys ${ }^{\circledR}$ NOVA-VEP Vision Testing System is a part of the Diopsys ${ }^{\circledR}$ NOVA suite of tests which use VEP to objectively measures the functional responses of the entire visual pathway from the anterior segment of the eye to the visual cortex. The intention of the design is to help improve sensitivity and specificity in diagnosing visual pathway disorders when used in conjunction with other diagnostic tests. The Diopsys ${ }^{\circledR}$ NOVA-VEP system comes equipped with two protocols - the Diopsys ${ }^{\circledR}$ NOVA-LX and Diopsys ${ }^{\circledR}$ NOVA-TR. 
Figure 1. The Diopsys VEP

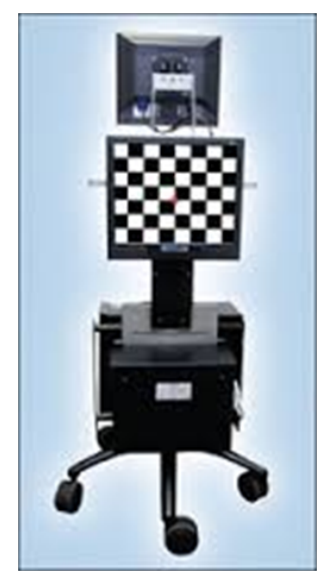

Figure 2. Diopsys VEP report showing normal (green) short and long latencies

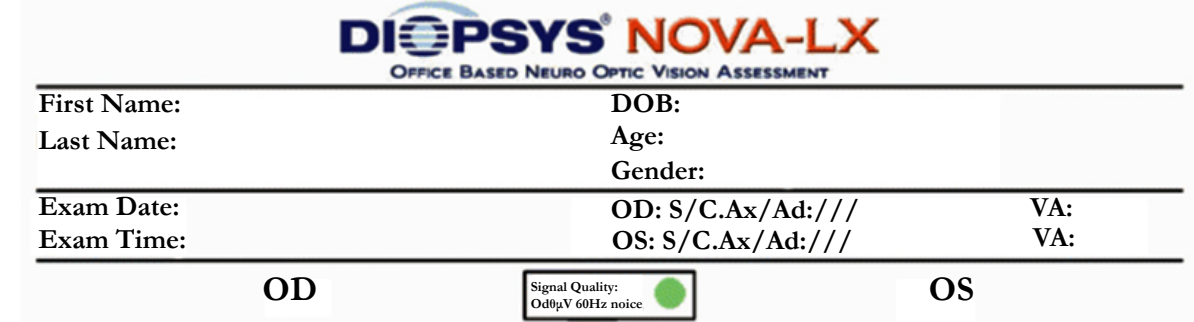
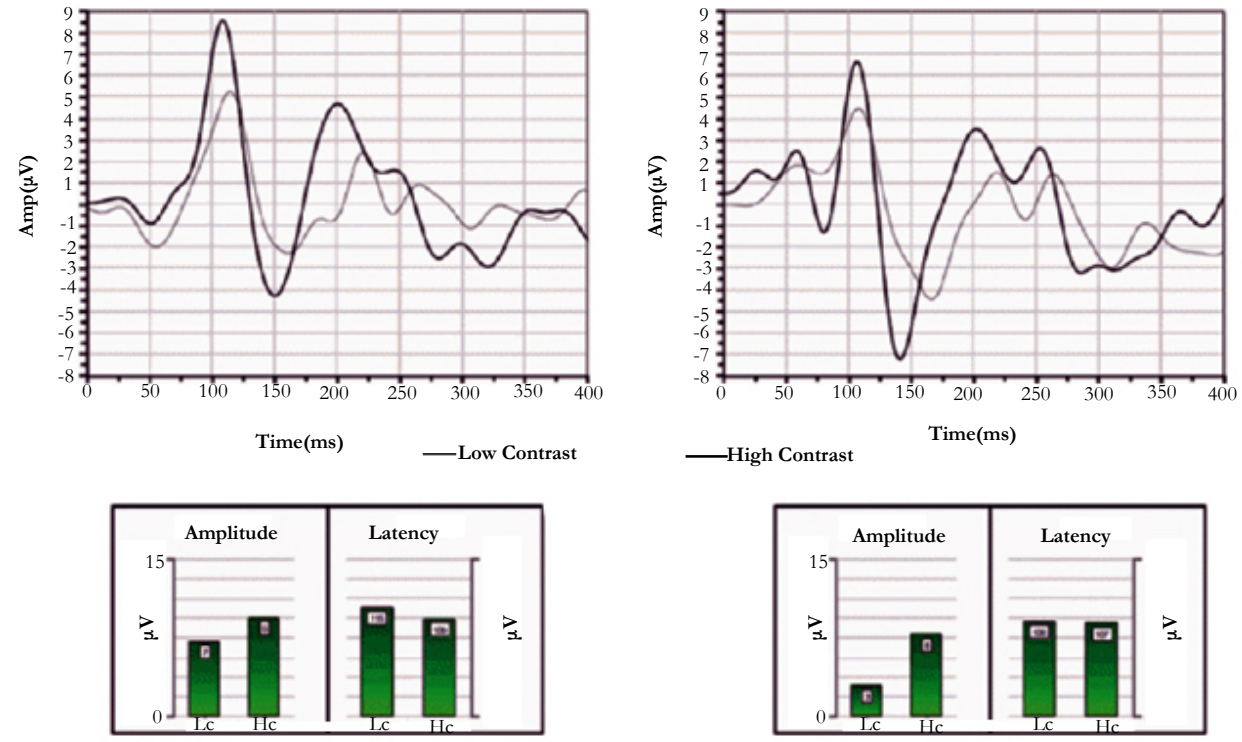

\begin{tabular}{|c|c|c|c|}
\hline Perametrs & OD & OS & Difference \\
\hline Amplitute Low Contrast $\mu \mathrm{V}$ & 7.2 & 3.0 & 4.2 \\
\hline Amplitute High Contrast $\mu \mathrm{V}$ & 9.4 & 7.9 & 1.5 \\
\hline Latency Low Contrast ms & 115.2 & 108.4 & 6.8 \\
\hline Latency High Contrast ms & 109.4 & 107.4 & 2.0 \\
\hline
\end{tabular}
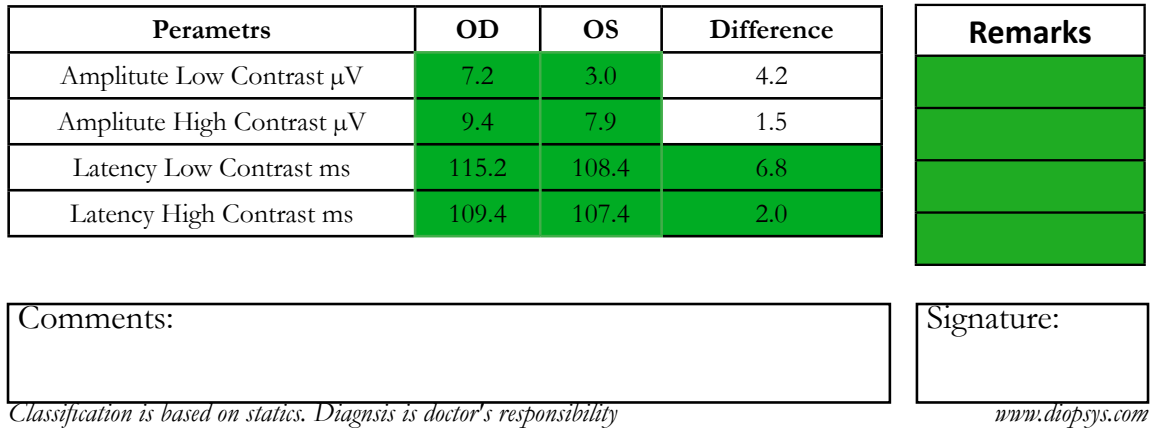

Copyright@2012 Diopsys, Inc. All Rights are Reserved. Softwate Version;2.14.2411-VX 
Figure 3. The EvokeDx (Konan Medical) is a relative compact self-contained image screen and processor

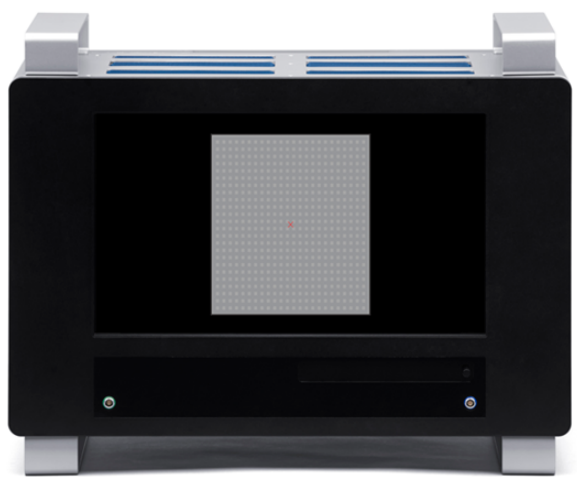

Figure 4. EvokeDx report. Multivariate analytics distill the complex VEP waveform into more understandable results

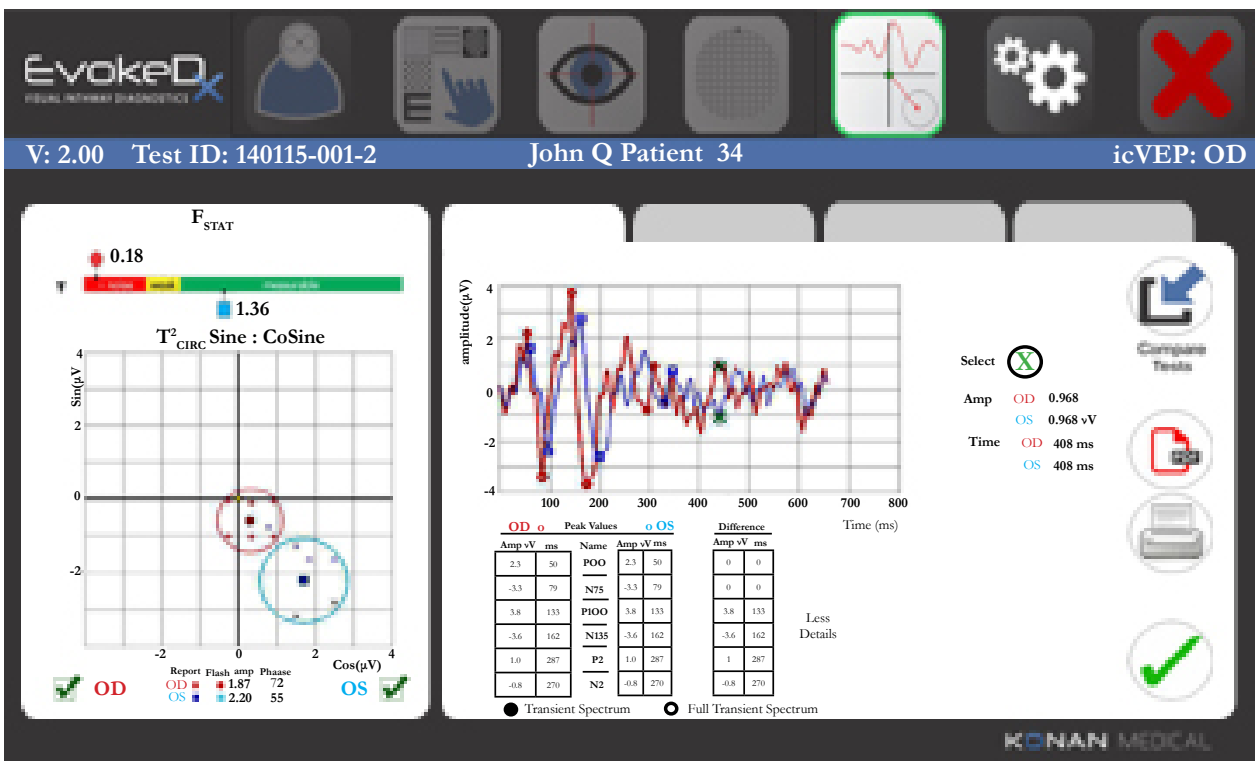

The Diopsys ${ }^{\circledR}$ NOVA-LX was designed to provide doctors with an easy-to-follow, advanced protocol that guides the technician through the test procedure. The color-coded reports show results for low contrast tests, indicating the health of magnocellular pathways, and high contrast tests, indicating the health of parvocellular pathways. The Diopsys ${ }^{\circledR}$ NOVA-TR was designed to allow the user to customize the stimulus to the patient and pathology, including the pattern, pattern size and contrast level.

The EvokeDx (Konan Medical) is Konan's patented Isolated Check VEP (icVEPTM) is a steady state, low contrast stimulus pattern that is thought to specifically target the magnocellular on pathway. The icVEP stimulus is presented as multiple, short runs (instead of a single, long run), enabling more reliable data collection while making the test easier on the patient.

SBIR Glaucoma investigation, Phase I (Columbia) and Phase II (Yale, Hamilton Eye Institute, University of Tennessee, and University Alabama, College of Optometry), reported 94\% and $92 \%$ accuracy respectively in separating glaucomatous from non-glaucoma patients using the patented icVEP protocol. An independent assessment in June 2013 in Beijing (TongRen and YouYi) report equivalent findings using icVEP: "high accuracy demonstrates the value as an aid in the diagnosis of open angle Glaucoma."'[10]

\section{Discussion}

Ever since visually evoked cortical potentials were first used as a diagnostic aid the important question has been whether they could detect visual field defects. In earlier investigations [11], light-flash stimulators illuminating the entire retina were used and the bioelectrical responses from both hemispheres were compared. Because asymmetries between the hemispheres were also found in normal people only differences of 50 percent or more between the responses of the right and left hemisphere were considered significant.

Later, methods of stimulating the temporal and nasal parts of the retina separately with flash and checkerboard stimulation were introduced. Finally, a sophisticated method of separating the signals from retinal areas stimulated simultaneously was devised.

Both the topographical position and the dimension and degree of the diminished sensitivity of the visual field are important for changes in the evoked potentials, the nearer to the center the visual field defect is localized, the larger the changes of the VEPs are expected to be. Thus a small relative scotoma located near the center may affect significant changes on the VEP while a large absolute scotoma in the periphery may cause only minor changes in VEP [12].

The larger latency increments have been reported when measured in eyes with large field defects [13] but there was no direct relation between field size and latency. The visual field may be nearly intact with a definite increase of latency in the affected eye. 
Increased pattern VEP latency was significantly correlated with both the severity and location of visual field defects and the degree of cupping and pallor of the optic disc in another study [14].

The VEP changes observed by some authors in the form of prolonged P100 latency were consistent with the central visual field defects qualitatively and quantitatively [15]. Therefore, it was concluded that the latency of P100 can be a useful quantitative index in the evaluation of glaucomatous visual function damage. The difference in diagnostic sensitivity to glaucoma between VEP and visual field changes were studied and the authors have suggested that combination of the two may be a more useful index.

\section{Summary}

As seen with any new set of data introduced to a previously accepted clinical standard, the implementation of VEP instrumentation in to common clinical setting is still finding its way. Making clinical decisions based of VEP data may often challenge accepted decision making based upon IOP threshold visual fields and OCT imaging. Given the notion that VEP represents a reasonable objective quantitative means of assessment of the visual process, it seems likely that these tests or others that will likely follow become an integral part of glaucoma management protocols.

With current evidence that VEP may offer some reliable objective evidence of early functional change, one can only hope that robust scientific evidence paired with more technical advancement of these types of tools may help us get a bit closer.

\section{References}

[1]. Kothari R, Bokariya P, Singh S, Singh R (2012) Significance of Visual Evoked Potentials in the Assessment of Visual Field Defects in Primary Open-Angle Glaucoma. A Review. Neuroscience Journal, 2013.

[2]. Pillai C, Ritch R, Derr P, Gonzalez A, Cox LK, et al. (2013) Sensitivity and Specificity of Short-Duration Transient Visual Evolked Potentials in Discriminating Normal From Glaucomatous Eyes. Invest Ophthalmol Vis Sci 54(4):2847-52.
[3]. Tello C, De Moraes GV, Prata TS (2010) Repeatability of short duration transient visual evoked potentials in normal subjects. Doc Ophthmol. 120:219-228.

[4]. Prata TS, Lima VC, De Moraes CGV (2012) Short duration transient visual evoked potentials in glaucomatous eyes. J Glaucoma 21:415-419.

[5]. Allingham RR, Damji KF, Shields MB (2011) Optic nerve, retina, and choroid. In: Allingham RR, Damji KF, Freedman S, Moroi SE, Rhee DJ, eds. Shields Textbook of Glaucoma. Philadelphia: Wolters Kluwer Health/Lippincott Williams \& Wilkins 51-81.

[6]. Mitchell KW, Wood CM, Howe JW, Church WH, Smith GTH, et al. (1989) The visual evoked potential in acute primary angle closure glaucoma. Br J Ophthalmol 73:448-456.

[7]. Morgan JE (1994) Selective cell death in glaucoma: does it really occur? $\mathrm{Br}$ J Ophthalmol 78:875-880.

[8]. Weber AJ, Chen H, Hubbard WC, Kaufman P (2000) Experimental glaucoma and cell size, density, and number in the primate lateral geniculate nucleus. Invest Ophthalmol Vis Sci 41: 1370-1379.

[9]. LC Bray, KW Mitchell, JW Howe, A Gashau (1992) "Visual function in glaucoma: a comparative evaluation of computerised static perimetry and the pattern visual evoked potential," Clinical Vision Sciences 7(1): 21-29.

[10]. Zemon V, Tsai JC, Forbes M, Al-Aswad LA, Chen CM, et al. (2008) Novel electrophysiological instrument for rapidand objective assessment of magnocellulardeficits associated with glaucoma. Doc Ophthalmol 117:233-243.

[11]. Vaughan HG, Katzman R (1964) "Evoked response in visual disorders," Annals of the New York Academy of Sciences1 12: 305-319.

[12]. Bartl G (1978) "The electroretinogram and the visual evoked potential in normal and glaucomatous eyes," GraefesArchiv fur Klinische und ExperimentelleOphthalmologie 207(4): 243-269.

[13]. Huber C, Wagner T (1978) "Electrophysiological evidence for glaucomatous lesions in the optic nerve," Ophthalmic Research 10(1): 22-29.

[14]. Towle VL, Moskowitz A, Sokol S, Schwartz B (1983) "The visual evoked potential in glaucoma and ocular hypertension: effects of check size, field size, and stimulation rate," Investigative Ophthalmology and Visual Science 24(2): 175-183.

[15]. He ZJ (1991) "The qualitative and quantitative diagnostic significance of PVEP in the evaluation of glaucomatous visual function damage," Zhonghua Yan KeZaZhi 27(1): 25-29.

Special Issue on
"Glaucoma and Hypertensive Retinopathy"
Edited by:
Dr. Pinakin Gunvant Davey, Western University of
Health Sciences, USA
E-mail: pdavey@westernu.edu

